\title{
Tailoring nomograms on patients and institutions: The utility of Fagan's two-step nomogram
}

\author{
Tommaso Prayer-Galetti*, Matteo Soligo, Massimo Iafrate and Fabrizio Dal Moro \\ Department of Surgery, Oncology and Gastroenterology, Urology Unit, University of Padua, Italy
}

\begin{abstract}
Besides oncologic outcomes, functional outcomes (preservation of potency and early recovery of the pre-operative urinary continence) are the two main goals of radical prostatectomy. Tailoring the most appropriate but also the most conservative surgery in patients with low or intermediate risk prostate cancer is one of the hottest topic in urologic oncology nowdays. A number of nomograms, risk calculators and graphic tools have been developed to gather data from biopsy, blood samples and radiologic imaging and help clinicians to find the best balance between oncologic and functional goals.
\end{abstract}

\section{Introduction}

Prostate cancer is the $2^{\text {nd }}$ more common invasive cancer diagnosed in men (26\% of all cancer diagnosis), and the 3rd more lethal, accounting for $9 \%$ of all cancer-related deaths [1]. The majority (80\%) of patients are diagnosed with a clinically localized disease, which is associated with a 100\% 5-year overall survival following the gold standard surgical intervention (radical prostatectomy + nerve-sparing technique + extended lymphadenectomy) [2]. Though these data look encouraging, $20-40 \%$ of patients will experienced a biochemical within 5 years following surgery, and up to $10 \%$ will progress to a clinically detectable recurrence [3]. Preservation of urinary and sexual function are additional concerns, since $20 \%$ of patients won't recover full urinary continence, and 20\%-78\% won't recover full potency [4,5].

\section{Neurovascular bundle preservation and risk of extracapsular invasion}

The exact anatomic location of prostatic neurovascular bundles was first described by Walsh and Donker in 1982 [6] however, nearly 30 years were needed to associate nerve-sparing surgery to a better preservation of sexual potency [7]. Since then, a number of nervesparing approaches (ie anterograde versus retrograde) and techniques (interfascial vs intrafascial) has been developed, and always been associated to better functional outcomes than non-nerve-sparing surgery [8]. However, rates of potency and continence recovery following surgery still vary, due to many influencing factors besides nerve-sparing approaches themselves (ie patient characteristics, surgeon experience, methods used to collect and report data) [9].

Extension of prostate cancer (PCa) beyond the gland pseudocapsule (extracapsular extension, ECE+ versus ECE-) has been associated to lower rate of disease-free recurrence and cancer-specific survival $[10,11]$. The ability to accurately identify ECE + may affect the likelihood to preserve neurovascular bundles [12]. Therefore, a comprehensive understanding of prostate anatomy, tumor burden and prostate cancer location within the gland is of utmost importance during pre-operative planning, in order to find the best balance between oncologic and functional goals.

\section{Clinical use of Fagan's two-step nomogram}

In the last years, literature has seen a flourishing of novel proposals of nomograms or risk calculators for ECE+ disease, alongside more traditional tools such as the aforementioned Partin Tables [13] and Cancer of the Prostate Risk Assessment (CAPRA) score [14]. Moreover, the additional predictive power given by magnetic resonance imaging (MRI) in the pre-operative setting has to be considered, as shown by Morlacco et al. [15] Feng et al. [16] and Tay et al. [17] among the first.

Another way to address the same issue requires to focus on statistics, as in Dal Moro et al. which resumed an old-fashioned but user-friendly graphic calculator (Fagan's two-step nomogram) to predict the risk of $\mathrm{ECE}+$ vs ECE- at radical prostatectomy, given the number of positive lateral cores at biopsy [18].

The original Fagan nomogram was developed by Dr Fagan in 1975. Bayesian probability theory was used to build a graphic tool able to predict the post-test probability of an individual to have a clinical condition, given the pre-test probability of the individual having that condition and the observed test outcome [19]. As underlined by Caraguel et al. Fagan's nomogram was difficult to use at bedside, since manual calculation of the likelihood ratio of negative and positive test results is always needed. A solution to that problem was the development of Fagan's two-step nomogram, a more user-friendly tool requiring no calculations and no statistical competencies at all [20].

In the aforementioned paper by Dal Moro et al. 1083 patients which underwent a 24-core transrectal or transperineal prostate biopsy were screened for eligibility. The final analysis focused on 94 patients with low- or intermediate risk prostate cancer which underwent

${ }^{\star}$ Correspondence to: Tommaso Prayer Galetti, Department of Surgery, Oncology and Gastroenterology - Urology, University of Padova, Italy, Tel: +390498212720, Fax: +39.0498218758, E-mail: tommaso.prayer@aopd.veneto.it

Key words: Fagan's nomogram, prostate cancer, extracapsular invasion, neurovascular bundles, Partin Tables

Received: June 10, 2018; Accepted: June 14, 2018; Published: June 17, 2018 
radical prostatectomy. The primary aim of the study was to test the improvement in the prediction of ECE- according to Partin Tables given a particular condition at prostate biopsy (pericapsular cores uninvolved by $\mathrm{PCa}$ ). In conclusion, authors found that the post-test probability of having ECE- when all lateral cores (ie pericapsular cores) were negative was $14.1 \%$, with a substantial gain of $7.5 \%$ as compared to Partin estimates for ECE- (21.6\%) [18].

The idea that the number of biopsy positive cores and/or the percentage of core involved by cancer may be used as a proxy for tumor burden is not new [21,22]. Moreover, the importance of a more accurate prostate sampling for pre-operative staging is widely accepted [23]. Dal Moro et al. while confirming what was already well known in the previous literature, also highlighted the great utility of an inexpensive nomogram, which would allow a good prediction of extracapsular extension without the need for additional imaging, such as MRI. Currently available literature about the side-specific accuracy of ECE is somewhat controversial, and larger studies may not add sufficient evidence [24-26]. However, this study was designed exactly to bypass those limitations: cores were considered as involved or not involved as a whole. In fact, the unique aspect of this paper is that only pericapsular cores (so, those more informative for gland invasion) were considered, and this may explain the improvement in the test sensibility and specificity $(91.7 \%$ and $45.4 \%$, respectively, in case of negative pericapsular cores) as compared to similar papers.

A number of papers have already investigated whether other preoperative clinical and pathological variables may be used alongside biopsy cores (either considered as "number of positive cores", "percentage of positive cores" or "percentage of core involved by cancer") may be used to improve the predictive ability for ECE+. Graefen et al. reported that the number of biopsy cores with high grade cancer, the number of positive biopsies and serum PSA may be independent predictors for ECE-; positive predictive values were higher than $82 \%$ in the lower risk groups ( $<1$ biopsy core with Gleason Score 4 or $5 \mathrm{PCa}$ and PSA $<10 \mathrm{ng} / \mathrm{mL}$, or $<1$ biopsy core with Gleason Score 4 or $5 \mathrm{PCa}$ and PSA $\geq 10 \mathrm{ng} / \mathrm{mL}$ ) [27]. Similar findings were reported by Sebo et al (percent of positive cores, serum PSA at biopsy and clinical Gleason Score), [28] Egawa et al. (number of cores involved by cancer, maximum cancer length, serum PSA at biopsy, clinical stage and clinical Gleason Score), [29] Goto et al. (total length of cancer in all biopsy cores, percent of poorly-differentiated cancer in each specimen, pre-biopsy serum PSA) [30] and Wills et al. (numbers of cores involved by cancer and highest clinical Gleason Score for the whole cohort; PCa bilaterality and serum PSA at subset multivariable analysis) [31].

However, none of these papers has focused on the well-known differences between institutions in biopsy approaches, biopsy templates and specimen reading. Due to their multifactorial origin, all these confounders will always be difficult to standardize, and may affect the validity of any nomogram and risk calculator using biopsy data. On the contrary, Dal Moro et al. have offered a clever clue to overcome these methodological differences, since Fagan's two step nomogram relies on diagnostic sensitivity and specificity of each institution's "test" for the target condition. This means that every healthcare centre, using its own local data, may tailor its own risk calculator for ECE+ vs ECE-, given a minimum diagnostic accuracy to be granted.

\section{Conclusions}

Fagan's two-step nomogram may be a flexible and interesting solution to predict the PCa pathologic feature of choice on the basis of the most appropriate biopsy, serum or imaging data. As underlined by Dal Moro et al. this risk calculator may easily adapt standardized nomograms to the clinical routine of each healthcare centre, thus stressing the uniqueness of the local experience (either surgical, radiological or anatomo-pathological), while maintaining the scaffold of validated tools. Though enough user-friendly to be drawn on paper, digital versions may be even more more handly to be used in the daily clinical practice.

\section{Acknowledgments}

All authors took part in writing the manuscript and approved the final, submitted version.

\section{Disclosure of potential conflicts of interest}

The authors have no competing interests to disclose.

\section{References}

1. Siegel RL, Miller KD, Jemal A (2015) Cancer statistics, 2015. CA Cancer J Clin 65: 5-29. [Crossref]

2. Cornford P, Bellmunt J, Bolla M, Briers E, De Santis M, et al. (2017) EAU-ESTROSIOG Guidelines on Prostate Cancer. Part II: Treatment of Relapsing, Metastatic, and Castration-Resistant Prostate Cancer. Eur Urol 71: 630-642. [Crossref]

3. Beresford MJ, Gillatt D, Benson RJ, Ajithkumar T (2010) A systematic review of the role of imaging before salvage radiotherapy for post-prostatectomy biochemical recurrence. Clin Oncol (R Coll Radiol) 22: 46-55. [Crossref]

4. Ficarra V, Borghesi M, Suardi N, De Naeyer G, Novara G, et al. (2013) Long-term evaluation of survival, continence and potency (SCP) outcomes after robot-assisted radical prostatectomy (RARP). BJU Int 112: 338-345. [Crossref]

5. Yuh B, Wilson T, Bochner B, Chan K, Palou J, et al. (2015) Systematic review and cumulative analysis of oncologic and functional outcomes after robot-assisted radical cystectomy. Eur Urol 67: 402-422. [Crossref]

6. Walsh PC, Donker PJ (1982) Impotence following radical prostatectomy: insight into etiology and prevention. J Urol 128: 492-497. [Crossref]

7. Walsh PC (1998) Anatomic radical prostatectomy: evolution of the surgical technique J Urol 160: 2418-2424. [Crossref]

8. Wang X, Wu Y, Guo J, Chen H, Weng X, et al. (2018) Intrafascial nerve-sparing radical prostatectomy improves patients' postoperative continence recovery and erectile function 29: e11297.

9. Ficarra V, Novara G, Rosen RC, Artibani W, Carroll PR, et al. (2012) Systematic Review and Meta-analysis of Studies Reporting Urinary Continence Recovery After Robot-assisted Radical Prostatectomy. Eur Urol 62: 405-417. [Crossref]

10. Mikel Hubanks J, Boorjian SA, Frank I, Gettman MT, Houston Thompson R, et al (2014) The presence of extracapsular extension is associated with an increased risk of death from prostate cancer after radical prostatectomy for patients with seminal vesicle invasion and negative lymph nodes. Urol Oncol Semin Orig Investig 32: 26. e1-26. e7. [Crossref]

11. Ploussard G, Agamy MA, Alenda O, Allory Y, Mouracade P, et al. (2011) Impact of positive surgical margins on prostate-specific antigen failure after radical prostatectomy in adjuvant treatment-naïve patients. BJU Int 107: 1748-1754. [Crossref]

12. Walz J, Epstein JI, Ganzer R, Graefen M, Guazzoni G, et al. (2016) A Critical Analysis of the Current Knowledge of Surgical Anatomy of the Prostate Related to Optimisation of Cancer Control and Preservation of Continence and Erection in Candidates for Radical Prostatectomy: An Update. Eur Urol 70: 301-311. [Crossref]

13. Eifler JB, Feng Z, Lin BM, Partin MT, Humphreys EB, et al. (2013) An updated prostate cancer staging nomogram (Partin tables) based on cases from 2006 to 2011. BJU Int 111: 22-29. [Crossref]

14. Cooperberg MR, Pasta DJ, Elkin EP, Litwin MS, Latini DM, et al. (2005) The University of California, San Francisco Cancer of the Prostate Risk Assessment score: a straightforward and reliable preoperative predictor of disease recurrence after radical prostatectomy. J Urol 173: 1938-1942. [Crossref]

15. Morlacco A, Sharma V, Viers BR, Rangel LJ, Carlson RE, et al. (2017) The Incremental Role of Magnetic Resonance Imaging for Prostate Cancer Staging before Radical Prostatectomy. Eur Urol 71: 701-704. [Crossref] 
16. Feng TS, Sharif-Afshar AR, Wu J, Li Q, Luthringer D, et al. (2015) Multiparametric MRI Improves Accuracy of Clinical Nomograms for Predicting Extracapsular Extension of Prostate Cancer. Urology 86: 332-337. [Crossref]

17. Tay KJ, Gupta RT, Brown AF, Silverman RK, Polascik TJ (2016) Defining the Incremental Utility of Prostate Multiparametric Magnetic Resonance Imaging at Standard and Specialized Read in Predicting Extracapsular Extension of Prostate Cancer. Eur Urol 70: 211-213. [Crossref]

18. Dal Moro F, Prayer-Galetti T, Angelini L, Vianello F, Gardiman M, et al. (2018) Study of diagnostic accuracy of Fagan's two-step nomogram in increasing the value of predictive tools for prostate cancer: application of specific spatial distribution of positive/negative bioptic cores to predict extracapsular extension. Aging Clin Exp Res. [Crossref]

19. Fagan TJ (1975) Letter: Nomogram for Bayes theorem. N Engl J Med 293: 257. [Crossref]

20. Caraguel CG, Vanderstichel R (2013) The two-step Fagan's nomogram: ad hoc interpretation of a diagnostic test result without calculation. Evid Based Med 18: 125128. [Crossref]

21. Park JW, Koh DH, Jang WS, Cho KS, Ham WS, et al. (2018) Predictors of adverse pathologic features after radical prostatectomy in low-risk prostate cancer. $B M C$ Cancer 18: 545. [Crossref]

22. Sankin A, Tareen B, Lepor H (2009) Side-specific factors associated with extracapsular extension and seminal vesicular invasion in men undergoing open radical retropubic prostatectomy. Prostate Cancer Prostatic Dis 12: 204-208. [Crossref]

23. Singh H, Canto EI, Shariat SF, Kadmon D, Miles BJ, et al. (2004) Six Additional Systematic Lateral Cores Enhance Sextant Biopsy Prediction of Pathological Features at Radical Prostatectomy. J Urol 171: 204-209. [Crossref]
24. Elliott SP, Shinohara K, Logan SL, Carroll PR (2002) Sextant Prostate Biopsies Predict Side and Sextant Site of Extracapsular Extension of Prostate Cancer. J Urol 168: 105109. [Crossref]

25. Frota R, Stein RJ, Turna B, Kamoi K, Lin YC, et al. (2009) Are prostate needle biopsies predictive of the laterality of significant cancer and positive surgical margins? BJU Int 104: 1599-1603. [Crossref]

26. Taneja SS, Penson DF, Epelbaum A, Hander T, Lepor He (1999) Does site specific labeling of sextant biopsy cores predict the site of extracapsular extension in radical prostatectomy surgical speciment? J Urol 162: 1352-1357.

27. Graefen M (2001) A validated strategy for side specific prediction of organ confined prostate cancer: a toool to select for nerve sparing radical prostatectomy. J Urol 165: 857-863.

28. Sebo TJ, Bock BJ, Cheville JC, Lohse C, Wollan P, et al. (2000) The percent of cores positive for cancer in prostate needle biopsy specimens is strongly predictive of tumor stage and volume at radical prostatectomy. J Urol 163: 174-178. [Crossref]

29. Egawa S, Suyama K, Matsumoto K, Satoh T, Uchida T, et al. (1998) Improved predictability of extracapsular extension and seminal vesicle involvement based on clinical and biopsy findings in prostate cancer in Japanese men. J Urol 52: 433-440. [Crossref]

30. Goto Y, Ohori M, Scardino PT (1998) Use of systematic biopsy results to predict pathologic stage in patients with clinically localized prostate cancer: A preliminary report. Int J Urol 5: 337-342. [Crossref]

31. Wills ML, Sauvageot J, Partin AW, Gurganus R, Epstein JI (1998) Ability of sextant biopsies to predict radical prostatectomy stage. Urology 51: 759-764. [Crossref]

Copyright: (C2018 Prayer-Galetti T. This is an open-access article distributed under the terms of the Creative Commons Attribution License, which permits unrestricted use, distribution, and reproduction in any medium, provided the original author and source are credited. 